\title{
Mindfulness-Based Interventions for Addiction
}

\section{Joan Trujols ${ }^{1,2}$}

Published online: 28 May 2020

(C) Springer Science+Business Media, LLC, part of Springer Nature 2020
In the recent New England Journal of Medicine article on mind-body medicine by Dossett et al. (2020), the authors make some excellent points about the current state of mindbody medicine and its promising future. However, their unsubstantiated claim that mind-body techniques, such as mindfulness, "may not be helpful in the setting of substance use disorder" is inaccurate. In fact, there is a large and rapidly growing body of evidence supporting the efficacy of mindfulness-based interventions (MBIs) - such as mindfulness-based relapse prevention (MBRP; Bowen et al. 2010) and mindfulness-oriented recovery enhancement (MORE; Garland 2013) - for the treatment of substance use disorders (SUDs). For example, a meta-analysis showed that MBIs effectively reduce cravings for - and the use of psychoactive substances, and decrease stress in SUD patients (Li et al. 2017). Moreover, current research on MBIs for SUDs is moving beyond efficacy studies to focus on elucidating the cognitive, affective, and neural processes underlying the effects of these interventions (Priddy et al. 2018). Research is also underway to identify - consistent with a precision medicine approach - the best predictors of treatment outcomes (Schwebel et al. 2020). Finally, other studies are being carried out to address the implementation of MBIs in real-world settings to facilitate the adoption of this approach in routine clinical practice (Wilson et al. 2017).

Given the evidence cited above, it is clear that the claim that mind-body interventions such as MBIs may not be helpful in the treatment of SUDs provide unreliable and misleading

Joan Trujols

jtrujols@santpau.cat

1 Unitat de Conductes Addictives, Servei de Psiquiatria, Institut d'Investigació Biomèdica Sant Pau (IIB Sant Pau), Hospital de la Santa Creu i Sant Pau, Sant Antoni Maria Claret 167, 08025 Barcelona, Catalonia, Spain

2 Centro de Investigación Biomédica en Red de Salud Mental (CIBERSAM), Madrid, Spain information to the potential readers (e.g., health policy planners, clinical administrators).

\section{References}

Bowen, S., Chawla, N., \& Marlatt, G. A. (2010). Mindfulness-based relapse prevention for addictive behaviors: A clinician's guide. New York: Guilford Press.

Dossett, M. L., Fricchione, G. L., \& Benson, H. (2020). A new era for mind-body medicine. New England Journal of Medicine, 382(15), $1390-1391$.

Garland, E. L. (2013). Mindfulness-oriented recovery enhancement for addiction, stress, and pain. Washington, DC: NASW Press.

Li, W., Howard, M. O., Garland, E. L., McGovern, P., \& Lazar, M. (2017). Mindfulness treatment for substance misuse: A systematic review and meta-analysis. Journal of Substance Abuse Treatment, $75,62-96$.

Priddy, S. E., Howard, M. O., Hanley, A. W., Riquino, M. R., FribergFelsted, K., \& Garland, E. L. (2018). Mindfulness meditation in the treatment of substance use disorders and preventing future relapse: Neurocognitive mechanisms and clinical implications. Substance Abuse and Rehabilitation, 9, 103-114.

Schwebel, F. J., Korecki, J. R., \& Witkiewitz, K. (2020). Addictive behavior change and mindfulness-based interventions: Current research and future directions. Current Addiction Reports, 7(2), $117-124$

Wilson, A. D., Roos, C. R., Robinson, C. S., Stein, E. R., Manuel, J. A., Enkema, M., et al. (2017). Mindfulness-based interventions for addictive behaviors: Implementation issues on the road ahead. Psychology of Addictive Behaviors, 31(8), 888-896.

Publisher's Note Springer Nature remains neutral with regard to jurisdictional claims in published maps and institutional affiliations. 